\title{
Das salas de autopsias e microscopias para a vida: mentoria informal em um programa de residência médica de patologia
}

\author{
From autopsy rooms and microscopes to life: informal mentoring in a pathology medical residency program \\ Monique Freire Santana1,2,3,4 (D) moniquefreire20@gmail.com \\ Luiz Carlos de Lima Ferreira ${ }^{3,4,5}$ (D) ferreira.luiz@gmail.com
}

\section{RESUMO}

Introdução: Além de demorada, a mentoria em Medicina ocorre em vários níveis e é complexa, pois pode incluir várias combinações de ensino, prática clínica, atendimento ao paciente e pesquisa.

Relato de experiência: Trata-se de um relato de experiência individual de uma mentorada em um programa de residência médica em patologia do Hospital Universitário Getúlio Vargas, de Manaus, no Amazonas. Descrevem-se os benefícios individuais e coletivos de uma mentoria fora de um programa formal, orientada pelo discernimento e pela maturidade de relacionamento entre mentor e mentorada.

Discussão: Discentes de programa de residência médica podem se beneficiar sobremaneira, pessoal e profissionalmente, de mentorias bem conduzidas.

Conclusão: No relacionamento de mentoria, são imprescindíveis qualidades como respeito mútuo, discernimento e abnegação, para que o mentorado cresça e desenvolva uma identidade profissional independente, ainda que atrelada à relação de mentoria, e amadurecida, em seu pleno desenvolvimento, a fim de que ele seja um novo mentor e novos alunos sejam beneficiados.

Palavras-chave: Educação Médica; Mentores; Residência; Patologia.

\section{ABSTRACT}

Introduction: Mentoring in medicine is time-consuming on several levels and complex, as it can include various combinations of teaching, clinical practice, patient care, and research.

Experience report: This report is on the individual experience of a mentee in a medical residency program in Pathology at the Getúlio Vargas University Hospital (Manaus-AM). The individual and collective benefits of mentoring outside a formal program are described, guided by the discernment and maturity of the relationship between mentor and mentee.

Discussion: Medical residency program students can benefit greatly, both personally and professionally, from well-conducted mentoring.

Conclusion: In the mentoring relationship, qualities such as mutual respect, discernment, and selflessness are essential so that the mentee grows and develops a fully developed and independent professional identity, albeit bound to the mentoring relationship, in order for him become a new mentor and to benefit new students.

Keywords: Medical Education; Mentors; Residency; Pathology.

\footnotetext{
${ }^{1}$ Fundação Centro de Controle de Oncologia do Estado do Amazonas, Manaus, Amazonas, Brasil.

${ }^{2}$ Centro Universitário Fametro, Manaus, Amazonas, Brasil.

3 Universidade do Estado do Amazonas, Manaus, Amazonas, Brasil.

${ }^{4}$ Fundação de Medicina Tropical Dr. Heitor Vieira Dourado, Manaus, Amazonas, Brasil.

${ }^{5}$ Universidade Federal do Amazonas, Manaus, Amazonas, Brasil.
}

Editora: Lia Marcia Cruz da Silveira

Recebido em 26/02/21; Aceito em 24/03/21.

Avaliado pelo processo de double blind review. 


\section{INTRODUÇÃO}

Além de demorada, a mentoria em Medicina ocorre em vários níveis e é complexa, pois pode incluir várias combinações de ensino, prática clínica, atendimento ao paciente e pesquisa'. O mentor deve demonstrar consistentemente um caráter íntegro, honesto, confiável, pautado pela ética e moralidade, pois mentorear é um relacionamento complexo ${ }^{2}$. A capacidade de identificar qual o melhor caminho para o pupilo, independentemente de seu desejo pessoal, de forma altruísta, é outra característica marcante de um bom mentor ${ }^{3}$.

Na residência médica, é perceptível pelos residentes a importância de ter um mentor, pois, com a relação de mentoria, ampliam-se as chances de excelente preparação para a carreira profissional $^{4}$. A orientação informal e formal por pares pode aumentar o bem-estar dos médicos residentes e proporcionar o desenvolvimento de competências para suas carreiras ${ }^{5}$.

O objetivo do relato é descrever como uma profícua experiência individual de mentoria em um programa de residência médica ( $\mathrm{PRM}$ ) pode ser singularmente proveitosa para o mentorado, com resultados extensivos a incontáveis acadêmicos de graduação e pós-graduação.

\section{RELATO DE EXPERIÊNCIA}

Sou médica, graduada pela Universidade do Estado do Amazonas (UEA) e, em 2014, prestei concurso para o PRM em patologia, iniciando minhas atividades em 2015. Nele, conheci o supervisor do programa, professor titular da Universidade Federal do Amazonas (Ufam), um patologista extremamente dedicado e qualificado, que com naturalidade e sabedoria conduziu-me em mentoria informal desde então. Sendo a única residente do serviço, tive o privilégio de acompanhar os preceptores em todas as atividades ofertadas. Dada minha inclinação para a docência e pesquisa, vinda do convívio com exímios docentes na UEA, aproximamo-nos pelos interesses em comum. Poucos meses após o início da residência, ele me ofereceu a oportunidade de acompanhá-lo e por vezes substituí-lo em algumas aulas de graduação. Certamente os acadêmicos notaram a pouca experiência da novata, porém, graças à persistência e ao encorajamento do mentor, eu ganharia confiança e crescimento a cada atividade.

Uma das primeiras atribuições que precisei cumprir durante a residência foi a apresentação das sessões anatomoclínicas, de autopsias e casos clínicos, para o corpo clínico e demais residentes do Hospital Universitário Getúlio Vargas. Essa atividade já havia, durante a graduação, despertado em mim o encanto pela patologia, por influência de outro grande mestre, meu professor na graduação, que a apresentava de forma brilhante, na Fundação de Medicina Tropical Dr. Heitor Vieira Dourado (FMT-HVD). Por influência direta deles, ganhei profundo fascínio pela autopsia, o que trouxe sessões anatomoclínicas memoráveis para os residentes.

Pesquisador nato, rapidamente meu mentor ofereceume a oportunidade de cursar o doutorado como aluna especial, durante a residência médica, no Programa de Pós-Graduação em Medicina Tropical da UEA em parceria com a FMT-HVD, expandindo ainda mais meus horizontes profissionais, com a oportunidade de participação de grupos de pesquisa da instituição. A partir de então, passamos a produzir cientificamente com seus pós-graduandos, mestrandos e doutorandos, agregando ainda mais alunos de iniciação científica e demais colaboradores interessados. Reuniões científicas, editais de financiamento e discussões de artigo foram boa parte do nosso dia a dia na residência médica. Um de nossos artigo publicados, meu trabalho de conclusão de curso, sobre erros em patologia ${ }^{6}$, originou em seguida um capítulo de livro ${ }^{7}$. Tivemos a oportunidade de aprovar um importante financiamento para três programas de pós-graduação, sendo dois do estado do Amazonas, por meio do Programa Nacional de Cooperação Acadêmica na Amazônia (Procad/Amazônia) da Coordenação de Aperfeiçoamento de Pessoal de Nível Superior (Capes), em 2018, como parte do meu projeto de doutorado. Vários trabalhos publicados em associação com outros grupos nacionais e internacionais, em diversas linhas de pesquisa além da patologia ${ }^{8,9,10,11}$, como acidentes por animais peçonhentos ${ }^{12,13,14}$, vírus da imunodeficiência humana/síndrome de imunodeficiência adquirida (human immunodeficiency virus/acquired immunodeficiency syndrome - HIV/Aids ${ }^{15}$ e, mais recentemente, coronavirus disease 2019 (Covid-19) ${ }^{16,17}$, além de trabalhos apresentados em congressos da especialidade e um grande número de estudantes de iniciação científica acompanhados por nós fazem parte dos frutos dessa mentoria.

Ainda durante a residência médica, meu mentor, então presidente da Comissão Estadual de Residência Médica do Amazonas (Cermam), incentivou-me a acompanhá-lo durantes as vistorias de programas de residência e a atuar como vistoriadora, avaliando programas de residência e emitindo pareceres com ele. Em vista dessa experiência, hoje sou coordenadora da Comissão de Residência Médica na instituição em que sou concursada como pesquisadora, a Fundação Centro de Controle de Oncologia do Amazonas (Fcecon-AM). Ainda na Cermam, tive a oportunidade de atuar como professora convidada da disciplina obrigatória de Bioestatística, ofertada anualmente aos residentes, de 2017 a 2020.

Em 2016, participamos do Encontro de Coordenadores dos Programas de Residência Médica em Patologia ${ }^{18}$, em que colaboramos na redação do documento final, o qual, em reuniões posteriores na diretoria da Sociedade Brasileira de 
Patologia (SBP), resultou no documento final proposto como Matriz Curricular da Residência Médica de Patologia ${ }^{19}$. Essa matriz foi apresentada por nós, em parceria com o secretáriogeral e a coordenadora de ensino da SBP, na plenária da Comissão Nacional de Residência Médica (CNRM) em Brasília, no ano seguinte, e aprovada em reunião, servindo de fundamento pedagógico para os PRMs. O início de uma participação ativa na SBP proporcionou-me a assessoria especial de graduação da atual diretoria da instituição, bem como parte na coordenação do Fórum de Ensino em Patologia (FEP).

Durante a residência e ao término dela, acompanhei-o em suas atividades de diagnóstico em laboratório privado e, depois de formada, tornei-me responsável técnica e patologista no serviço em que atualmente trabalho. Passei a ser professora de Patologia em uma Faculdade de Medicina privada, dando continuidade às atividades de docência, e mantive as aulas como professora convidada na Faculdade de Medicina da Ufam, na qual comecei a lecionar, a seu convite, como residente. Mais recentemente, durante a pandemia de Covid-19 em Manaus, no Amazonas, tive a oportunidade de realizar as autopsias completas dos pacientes, gerando até aqui duas publicações ${ }^{16,17}$, com outras em andamento, além de materiais didáticos para o FEP, disponibilizados em aulas ministradas por patologistas de todo o Brasil e acessíveis ao público por meio de seu canal no YouTube.

\section{DISCUSSÃO}

O objetivo deste relato de experiência é descrever como a relação de mentoria pode beneficiar o mentorado em diversos aspectos e como seus resultados positivos podem se estender a muitos outros alunos, direta e indiretamente. Não existe, no PRM relatado, nenhum registro de mentoria. Nessa experiência, ela foi estabelecida de forma espontânea. Não houve o processo de sistematização de uma mentoria, em que existe uma seleção prévia do mentor. Em pesquisa realizada com acadêmicos de Medicina, a procura e a escolha por seus mentores ocorrem durante estágios de internato, em atividades de pesquisa ou com base em interesses semelhantes ${ }^{20}$. No caso descrito, certamente os interesses em comum, como a própria especialidade médica, e as afinidades por docência e pesquisa exerceram influência na aproximação mútua entre mentor e mentorada.

A despeito de haver grande progresso na elaboração das novas matrizes de competência dos PRMs na CNRM, incluindo a da patologia, pouca ênfase tem sido dada ao processo de formação de mentorias nessa importante etapa da educação médica. Durante os primeiros estágios de uma carreira, a mentoria está associada a uma alta satisfação e pode orientar o desenvolvimento de uma experiência profissional bemsucedida ${ }^{4}$. No entanto, muitas vezes não é abordada ativamente durante o treinamento, o que pode afetar a formação e as competências do residente ${ }^{21,22}$.

O termo mais comumente utilizado pela CNRM para descrever os responsáveis diretos pela formação dos residentes é "preceptor/supervisor"23. Mentor ${ }^{22,24}$ é um termo utilizado por aquele que se relaciona com o jovem mentorado fora de seu ambiente imediato de prática profissional, com as funções de guiar, orientar e aconselhar na realização de objetivos pessoais, buscando o desenvolvimento interpessoal, psicossocial, educacional e o estímulo ao raciocínio crítico. A mentoria pode se desenvolver de forma coletiva ou particular, e até mesmo confidencial, de forma atemporal. Não tem função avaliativa, representando uma forma de transferência de conhecimento de um profissional mais experiente e maduro para um residente neófito, em formação, capacitando-o para a tomada de decisões. O mentor exerce um papel importante na formação do residente ao ensiná-lo a reconhecer suas fraquezas e virtudes profissionais, influenciando e aconselhado em sua vida pessoal, incluindo aspectos relacionados à espiritualidade $\mathrm{e}^{24-26}$.

Esse relacionamento pode ocorrer naturalmente, como uma atividade poderosa para transmitir sabedoria e conhecimento do sábio para o aluno, resultando em sucesso do pupilo e realização do mentor ${ }^{27}$. A mentoria informal é o encontro natural de um mentor e um pupilo, baseado em um relacionamento de amizade, respeito pessoal e profissional, e na admiração de um pelo outro. Sendo assim, a mentoria formal difere da mentoria informal porque, na primeira, a instituição desenvolve um programa e um processo para que a mentoria ocorra $^{28}$. A despeito de ampla discussão sobre a satisfação e qualidade das mentorias informais, é certo que os programas de mentoria formal precisam refletir e absorver muitas das características da mentoria informal ${ }^{29}$. Neste relato, descrevemse a construção desse relacionamento e os benefícios de uma mentoria informal em um PRM, a despeito da ausência de um programa formal.

Evidências sugerem que algumas atitudes características e qualidades pessoais, conhecimentos, habilidades e comportamentos são comuns entre mentores de sucesso ${ }^{2}$. Relacionamentos de mentoria bem-sucedidos são caracterizados por reciprocidade, respeito mútuo, expectativas claras, conexão pessoal e valores compartilhados. Por sua vez, relacionamentos de mentoria fracassados foram caracterizados por comunicação deficiente, falta de compromisso, diferenças de personalidade, competição percebida (ou real), conflitos de interesse e inexperiência do mentor ${ }^{3}$. É inegável, dado o volume de tarefas em comum exercidas nessa mentoria, a quantidade de tempo despendida no exercício das atividades profissionais. Naturalmente, a conexão pessoal, o incentivo contínuo por parte do mentor e o compartilhamento de valores éticos em 
comum influenciaram no bom andamento da mentoria, contribuindo para a evolução no desempenho da mentorada. A ausência de qualquer sentimento de egoísmo, egocentrismo ou vaidade do mentor, orientado apenas pelo desejo altruísta de ver o desenvolvimento e crescimento de seu mentorado, é fundamental para que a relação entre ambos seja saudável. Certamente esse comportamento é perceptível nas inúmeras oportunidades acadêmicas e profissionais cedidas pelo mentor neste relato.

Mentores eficazes devem, de forma altruísta e abnegada, estar compromissados com o sucesso de seu mentorado, colocando-o acima de quaisquer considerações pessoais ${ }^{30}$. O mentor deve ter boa reputação e experiência suficiente para levar riqueza de conhecimentos, habilidades e recursos ao mentorado e demonstrar constantemente em seu caráter integridade, honestidade, confiabilidade, ética e moralidade ${ }^{20}$. Um dever essencial do mentor é criar um ambiente seguro e não ameaçador, para que o mentorado se sinta livre para expressar seus sentimentos, suas preocupações e opiniões interiores ${ }^{21}$, especialmente em contextos delicados, como depressão e burnout ${ }^{21,30}$.

Um dos principais desafios para mentores e pupilos é a falta de tempo. Os participantes de pesquisa conduzida por Straus et al. ${ }^{3}$ afirmaram que mentores eficazes garantiram que permanecessem acessíveis aos seus pupilos, mesmo localizados a distância. Embora eles possam não ser capazes de se encontrar em pessoa regularmente, mentores eficazes usavam e-mail e telefone para garantir a acessibilidade. Neste relato, devido às múltiplas atividades em comum exercidas pelos profissionais, foram necessárias, durante o período de distanciamento físico na pandemia de Covid-19, a utilização de serviços de conferência remota para discussão de casos de patologia cirúrgica, autopsia e atividades de pesquisa.

Em um relacionamento de mentoria, naturalmente as redes de influência profissional de colegas e colaboradores beneficiam diretamente o mentorado ${ }^{3}$, facilmente percebidas neste relato por meio de sua inserção em diferentes atividades de pesquisa e ensino, que repercutiram positivamente em suas atuais atuações profissionais. As oportunidades de publicações e a orientação do trabalho de conclusão de curso são bastante valorizadas em uma mentoria ${ }^{30,31}$. Os residentes procuram também aconselhamento na tomada de decisões futuras, e o mentor geralmente atua como um guia de carreira, fellowship, orientador de subáreas de PRM ou na pós-graduação stricto sensu. Para o recém-formado, o mentor é um grande auxílio na implantação de laboratórios de patologia e na compra de materiais básicos em início de carreira, além de um conselheiro em longo prazo ${ }^{32,33}$.

Aagaard et al. ${ }^{20}$ descrevem as múltiplas funções que podem ser desempenhadas por um mentor: apoio pessoal (motivação, apoio moral, conselho pessoal); aconselhamento de carreira (auxiliando nas decisões de escolha de carreira e no avanço nela); modelagem de papel para carreira e família; e colaboração em pesquisas/projetos. Tais atribuições podem ser facilmente percebidas no relacionamento aqui descrito entre mentor e mentorada. Indubitavelmente, a abrangência holística da mentoria pode ser percebida nas diferentes formas de influência que o mentor exerce ao longo do tempo de mentoria, mantendo e respeitando a individualidade e o desenvolvimento da mentorada. O bom mentor é acessível e capaz de identificar e apoiar o desenvolvimento de potenciais forças e habilidades em seus pupilos ${ }^{3}$. Na relação de mentoria descrita, não houve qualquer exigência ou intimidação para o cumprimento de atividades, apenas a presença de um estímulo constante e recíproco ao crescimento e desenvolvimento mútuos.

Sabidamente, há benefícios diretos para o mentor nas mentorias: o desenvolvimento de habilidades de comunicação, ensino e liderança, bem como a satisfação pessoal. Para o mentorado, os benefícios são abrangentes: a possibilidade de desenvolvimento pessoal e profissional por meio de um feedback construtivo e observação de um modelo positivo, o desenvolvimento de habilidades de comunicação, a orientação de carreira e de possibilidades de socialização dentro dela, além de oportunidades de pesquisa ${ }^{34}$. Neste relato, pode-se observar que os benefícios da mentoria ultrapassam a relação entre as duas pessoas envolvidas. Na mentoria, há muitos personagens de diferentes ambientes acadêmicos, o que expande os horizontes dos muitos discentes de graduação e pós-graduação envolvidos.

Nossa experiência de mentoria se desenvolveu de forma exitosa, visto que a mentorada adquiriu, além dos conhecimentos necessários para um médico especialista, as habilidades acadêmicas e científicas almejadas. A mentoria relatada demonstra que, quando esse relacionamento é exercido entre mentor e residente dedicados e compromissados, as chances de êxito profissional são potencializadas.

\section{CONCLUSÃO}

Diversos benefícios individuais e coletivos foram contemplados neste breve relato, e esperamos que ainda mais discentes possam se beneficiar desse exemplo de mentoria a partir de um PRM. A despeito da tentativa de impessoalidade adotada na redação deste relato de experiência, é praticamente impossível não imprimir sentimentos próprios. Mais do que um artigo científico, ele retrata o sentimento de gratidão a todos os mestres e em especial ao meu mentor. Esses profissionais da educação abdicam de parte de si, de seu tempo e de sua vida ao abnegadamente entregarem sua experiência para que 
seus alunos cresçam em conhecimento e sabedoria. Grande satisfação é a minha durante a docência e a pesquisa ao compartilhar com meus alunos os benefícios que pude obter de tão valorosa ancestralidade acadêmica e científica.

\section{CONTRIBUIÇÃO DOS AUTORES}

Monique Freire Santana concebeu, redigiu e revisou o manuscrito. Luiz Carlos de Lima Ferreira participou da redação e revisão do manuscrito.

\section{CONFLITO DE INTERESSES}

Declaramos não haver conflito de interesses.

\section{FINANCIAMENTO}

Declaramos não haver financiamento.

\section{REFERÊNCIAS}

1. Moutsopoulos HM. Mentoring in medicine. Eur J Clin Invest. 2019;49(8).

2. Geraci SA, Thigpen SC. A review of mentoring in academic medicine. Am J Med Sci. 2017;353:151-7. doi: 10.1016/j.amjms.2016.12.002.

3. Straus SE, Johnson MO, Marquez C, Feldman MD. Characteristics of successful and failed mentoring relationships: a qualitative study across two academic health centers. Acad Med. 2013;88:82-9.

4. Ramanan RA, Taylor WC, Davis RB, Phillips RS. Mentoring matters. J Gen Intern Med. 2006 Apr;21:340-5 [acesso em 11 mar 2021]. Disponível em: http://link.springer.com/10.1111/j.1525-1497.2006.00346.x.

5. Lorenzetti D, Pethrick $H$, Nowell L, Lorenzetti L, Jacobsen M, OddonePaolucci E. Mentorship in medical residency education: anatomy of a systematic review. Epub ahead of print 2020. doi: 10.4135/9781529714180.

6. Santana MF, Ferreira LCL. Diagnostic errors in surgical pathology. J Bras Patol Med Lab. 2017;53:124-29. doi: 10.5935/1676-2444.20170021.

7. Santana MF, Ferreira LCL. Errors in surgical pathology laboratory. In: Quality control in laboratory. Zaman GS (ed). InTech; 2018 [London] [acesso em 25 fev 2021]. Disponível em: http://www.intechopen.com/books/qualitycontrol-in-laboratory/errors-in-surgical-pathology-laboratory.

8. Palhares AEM, Ferreira L, Freire M, Castillo P, Martínez MJ, Hurtado JC, et al. Performance of the minimally invasive autopsy tool for cause of death determination in adult deaths from the Brazilian Amazon: an observational study. Virchows Arch. 2019 Nov 14;475:649-58. doi: 10.1007/s00428-01902602-z.

9. Santana MF, Ferreira LCL, de Oliveira JGN, Francesconi F. Histopathological changes in epithelium of hair follicles and acrosyringium caused by measles in child. An Bras Dermatol. 2020 Mar;95:238-40 [acesso em 25 fev 2021]. Disponível em: https://linkinghub.elsevier.com/retrieve/pii/ S0365059620300313.

10. Santana MF, João GAP, de Lacerda MVG, Ferreira LCL. Diabetes insipidus secondary to tuberculous meningoencephalitis with hypothalamic involvement extending to the hypophysis: a case report. Rev Soc Bras Med Trop. 2018 Dec;51:86-7 [acesso em 25 fev 2021]. Disponível em: http://www. scielo.br/scielo.php?script=sci arttext\&pid=S0037-86822018000600865\&ln $\mathrm{g}=\mathrm{en} \& \mathrm{t}$ Ing=en.

11. Santana MF, Ferreira LCL. Accuracy of thyroid fine needle aspiration and cyto-histologic correlation: a 5 year-experience. Paripex Indian J. Res. 2019;8.

12. Sachett JAG, Val FF, Alcântara JA, Cubas-Vega N, Montenegro CS, da Silva IM, et al. Bothrops atrox Snakebite: how a bad decision may lead to a chronic disability: a case report. Wilderness Environ Med. 2020 Sept;31:317-23 [acesso em 25 fev 2021]. Disponível em: https://linkinghub.elsevier.com/ retrieve/pii/S1080603220300557.
13. Ibiapina HNS, Costa AG, Sachett JAG, Silva IM, Tarragô AM, Neves JCF, et al. An immunological stairway to severe tissue complication assembly in Bothrops atrox Snakebites. Front Immunol. 2019 Aug 13;10 [acesso em 25 fev 2021]. Disponível em: https://www.frontiersin.org/article/10.3389/ fimmu.2019.01882/full.

14. Souza AS, Sachett JAG, Alcântara JA, Freire M, Alecrim MGC, Lacerda M, et al. Snakebites as cause of deaths in the Western Brazilian Amazon: why and who dies? Deaths from snakebites in the Amazon. Toxicon. 2018;145:15-24.

15. Gama WM, Oliveira LB, Chaves YO, Ribeiro F, Almeida TVR, Baptista $B J A$, et al. Increased levels of reactive oxygen species in platelets and platelet-derived microparticles and the risk of respiratory failure in HIV/AIDS patients. Mem Inst Oswaldo Cruz. 2020;115 [acesso em 25 fev 2021]. Disponível em: http://www.scielo.br/scielo.php?script=sci arttext\&pid=S0074-02762020000100416\&tlng=en.

16. Santana MF, Pivoto G, Alexandre MAA, Baía-da-Silva DC, Borba MGS, Val FA et al. Confirmed Invasive pulmonary aspergillosis and COVID-19: the value of postmortem findings to support antemortem management. Rev Soc Bras Med Trop. 2020;53 [acesso em 25 fev 2021]. Disponível em: http://www.scielo. $\mathrm{br} /$ scielo.php?script=sci_arttext\&pid=S0037-86822020000100719\&tlng=en .

17. Santana MF, Borba MGS, Baía-da-Silva DC, Val F, Alexandre MAA, BritoSousa JD, et al. Case report: adrenal pathology findings in severe COVID-19: an autopsy study. Am J Trop Med Hyg. 2020 Oct 7;103:1604-7. doi: 10.4269/ajtmh.20-0787.

18. Domingues MAC. Balanço: I Encontro de Coordenadores de Residência Médica em Patologia. Sociedade Brasileira de Patologia; 2017 [acesso em 25 fev 2021]. Disponível em: http://www.sbp.org.br/balanco-i-encontrode-coordenadores-de-residencia-medica-em-patologia/.

19. Matriz de Competências - Patologia. 2017 [acesso em 25 fev 2021] Disponível em: http://portal.mec.gov.br/index.php?option=com_do $\mathrm{cman} \&$ view $=$ download\&alias $=102681$ - matriz-patologia\&category slug=novembro-2018-pdf\&ltemid=30192.

20. Aagaard EM, Hauer KE. A cross-sectional descriptive study of mentoring relationships formed by medical students. J Gen Intern Med. 2003;18:298-302.

21. Esposito MJ, Roychoudhury S, Fornari A. A professionalism and mentoring curriculum for pathology residents in training. Acad Pathol. 2018;5:237428951880506.

22. Shanafelt TD, West C, Zhao X, Novotny P, Kolars J, Habermann T, et al. Relationship between increased personal well-being and enhanced empathy among internal medicine residents. J Gen Intern Med. 2005 July;20:559-64 [acesso em 25 fev 2021]. Disponível em: http://www.ncbi. nlm.nih.gov/pubmed/16050855.

23. Brasil. Resolução CNRM n 006/2004, de 9 de junho de 2004 [acesso em 25 fev 2021]. Disponível em: http://portal.mec.gov.br/sesu/arquivos/pdf/ cnrm_062004.pdf.

24. Botti SHO, Rego S. Preceptor, supervisor, tutor e mentor: quais são seus papéis? Rev Bras Educ Med. 2008;32:363-73.

25. Geraci SA, Thigpen SC. A review of mentoring in academic medicine. Am J Med Sci. 2017 Feb;353:151-7 [acesso em 25 fev 2021]. Disponível em: http://www.ncbi.nlm.nih.gov/pubmed/28183416.

26. Mills JE, Francis $\mathrm{KL}$, Bonner A. Mentoring, clinical supervision and preceptoring: clarifying the conceptual definitions for Australian rural nurses. A review of the literature. Rural Remote Health. 5(3):410.

27. Inzer LD, Crawford CB. A review of formal and informal mentoring processes, problems, and design. J Leadersh Educ. 2005;4-31-50 [acesso em 21 mar 2021]. Disponível em: https://journalofleadershiped.org/ jole_articles/a-review-of-formal-and-informal-mentoring-processesproblems-and-design/.

28. Ragins BR, Cotton JL, Miller JS. Marginal mentoring: the effects of type of mentor, quality of relationship, and program design on work and career attitudes. Acad Manag J. 2000;43:1177-94.

29. Clutterbuck D. Formal $v$ informal mentoring: time to shift the debate? 2005 [acesso em 21 mar 2021]. Disponível em: https:// scottishmentoringnetwork.co.uk/assets/downloads/resources/formal-vinformal-mentoring.pdf. 
30. Fornari A, Murray TS, Menzin AW, Woo VA, Clifton M, Lombardi M, et al. Mentoring program design and implementation in new medical schools. Med Educ Online. 2014 Jan 25;19:24570.

31. Straus SE, Chatur F, Taylor M. Issues in the mentor-mentee relationship in academic medicine: a qualitative study. Acad. Med. 2009 Jan;84:135-9.

32. Detsky AS, Baerlocher MO. Academic mentoring - how to give it and how to get it. JAMA. 2007 May 16;297:2134-6.
33. Berk RA, Berg J, Mortimer R, Walton-Moss B, Yeo TP. Measuring the Effectiveness of faculty mentoring relationships. Acad Med. 2005 Jan;80:66-71.

34. Nimmons D, Giny S, Rosenthal J. Medical student mentoring programs: current insights. Adv Med Educ Pract. 2019;10:113-23. 